\title{
GMSM: A Design Method for Misinformation-Aware Social Media
}

\author{
${\text { Malik Almaliki }{ }^{1} \text {, H. Hashim }}^{1}$, A. Alzighaibi ${ }^{1}$ \& E. Atlam ${ }^{1,2}$ \\ ${ }^{1}$ College of Computer Science and Engineering, Taibah University, Yanbu, KSA \\ ${ }^{2}$ Faculty of Science, Tanta University, Tanta, Egypt \\ Correspondence: Malik Almaliki, College of Computer Science and Engineering, Taibah University, Yanbu, \\ KSA.
}

Received: October 5, 2020

Accepted: October 15, 2020

Online Published: October 23, 2020

doi:10.5539/cis.v13n4p23

URL: https://doi.org/10.5539/cis.v13n4p23

\begin{abstract}
Misinformation is highly circulating on social media which harmfully affect users of these platforms and the online content value. Previous efforts to decrease misinformation distribution on social media have mainly focused on the development of misinformation detection algorithms. To extend these efforts, this paper adopts gamification techniques to minimize the spread of misinformation on social media and proposes a three-phase requirement engineering method for the design of a Gamified Misinformation-aware Social Media (GMSM). The method combines the strengths of well-known requirement engineering approaches in a sequence that offers software engineers better understanding of users' requirements on the adoption of gamification to minimize the spread of misinformation on social media. This can lead to a better coverage of important users' requirements thus, a better user satisfaction and a higher quality of online content.
\end{abstract}

Keywords: gamification, misinformation, social media, software engineering

\section{Introduction}

\subsection{Misinformation and Gamification}

Social media, such as Twitter, WhatsApp, Facebook, etc, are becoming largely adopted channels to generate and exchange users' online created information. They give users the power to create and share information in a quick, timely and user-friendly style. They can also act as powerful and informative means for rapid information exchange throughout disasters (Oh, Agrawal et al. 2013, Starbird, Maddock et al. 2014) and as a rich source for creating and sharing knowledge (Kim, Decker et al. 2010). Therefore, social media platforms have become an important and highly valuable information source (Chen and Sin 2013).

Nevertheless, a vital threat that confronts these platforms is the low quality and reliability of some of the created and shared information on them (Chen and Sin 2013). Misinformation, characterized as incorrect or imprecise information, is largely and rapidly increasing (purposely or accidentally) on those platforms (Karlova and Fisher 2013). This quick increase of online false information is perceived by The World Economic Forum as an important problem that should be addressed on an international level (Vis 2014). Additionally, statistics regarding online misinformation spread show that about $67 \%$ of users shared misinformation on social media. Furthermore, about $94 \%$ of users said that they encountered distributed misinformation on social media by other users (Chen and Sin 2013).

Misinformation is present on social media platforms and can appear in different forms such as urban, rumors, factoids, legends, etc (Karlova and Fisher 2013). Moreover, the circulation of misinformation on social media could cause serious confusion, anxiety and undesirable emotions to users (Budak, Agrawal et al. 2011). Misinformation can also facilitate online illegal actions that cause serious damage to users (Chen and Sin 2013). A recent study showed that one in every ten Americans has untrue information about them posted online and as a result, they experienced mental or emotional stress (see figure 1) (Duggan 2017). Furthermore, misinformation spread can highly damage the beneficial use of information and knowledge shared on social media (Budak, Agrawal et al. 2011). 


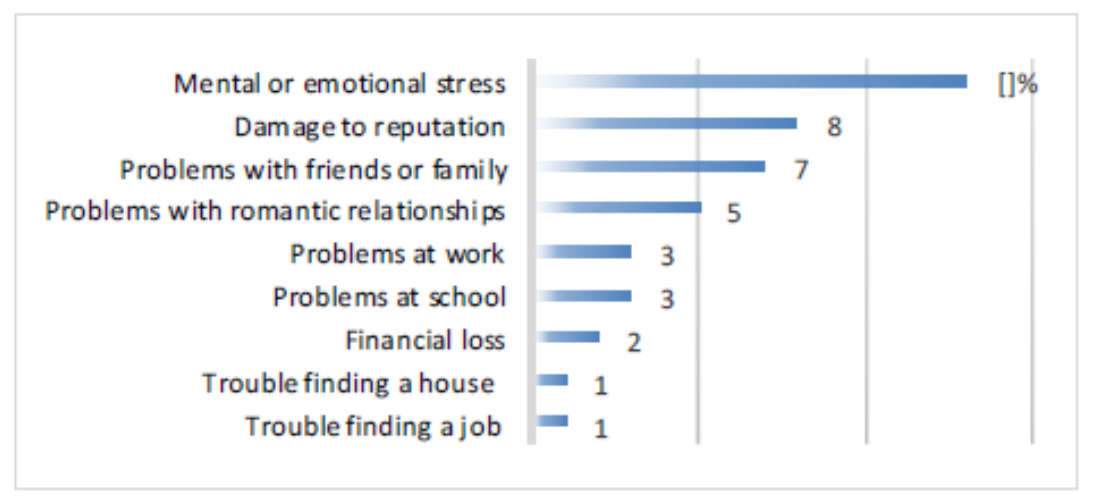

Figure 1. Forms of misinformation problems reported by US adults (Duggan 2017)

Efforts to lower the distribution of online misinformation have mainly focused on the development of algorithms that can detect false information (Ratkiewicz, Conover et al. 2010, Budak, Agrawal et al. 2011, Starbird, Maddock et al. 2014). Another research reported on the influence of user-intrinsic features (e.g., motivation and personality characteristic) on users' behavior towards misinformation distribution on social media (Chen 2016). To extend these efforts, our recent study (Almaliki 2019), proposed a conceptual framework for the use of gamification elements in the design phase of social media to minimize the distribution of misinformation related to news information shared by users (see figure 2) (i.e. information related to present or past incidents on various matters (e.g. war, politics, health, economy, business, etc.)).

Gamification refers to the use of game design elements (e.g. points, digital badges, leader-boards, etc.) in a non-game domain (Deterding, Dixon et al. 2011). Recently, gamification is being used as a powerful approach to alter users' attitudes towards a certain matter by maximizing their motivation and engagement (e.g. lower misinformation distribution) (Herzig, Ameling et al. 2012). A popular practice of gamification adoption is to adopt the scoring elements of games (e.g. levels, digital badges, etc) and use them in a non-game domain (e.g. the domain of education) (Nicholson 2019). Almaliki (2019) integrated Gamification into the design stage of social media platforms. This integration aims to improve users' perceptions regarding the harms caused by misinformation spread and increase their critical digital literacy. For instance, users could win or lose points based on the quality ratings given by their online friends on their news posts.

In (Almaliki 2019), an empirical investigation of users' perceptions towards the integration of a number of game design elements into social media to reduce the distribution of misinformation. The reported findings showed evident variations of users' perceptions that include: privacy, rewards and the rewarding method, social recognition, notification mechanism and UI design. The results advocate the need for novel and systematic engineering approaches to effectively employ gamification on social media for the purpose of minimizing the distribution of misinformation. Therefore, the conceptual framework (see figure 2) was proposed to help software engineers to integrate gamification into the design of social media.

However, one unanswered question can be, as software engineers, how would we adopt the proposed framework to successfully design a gamified misinformation-ware social media? To answer this question, this paper builds upon the previously devised conceptual model (see figure 2) and proposes GMSM, a three-phase requirement engineering method for the design of a Gamified Misinformation-aware Social Media. GMSM aims at providing software engineers with a clear approach for the design of a gamified misinformation-aware social media. The rest of this paper is organized as follows. Section 2 and its sub-sections introduce and discuss the proposed method and Section 3 contains the paper's conclusion. 


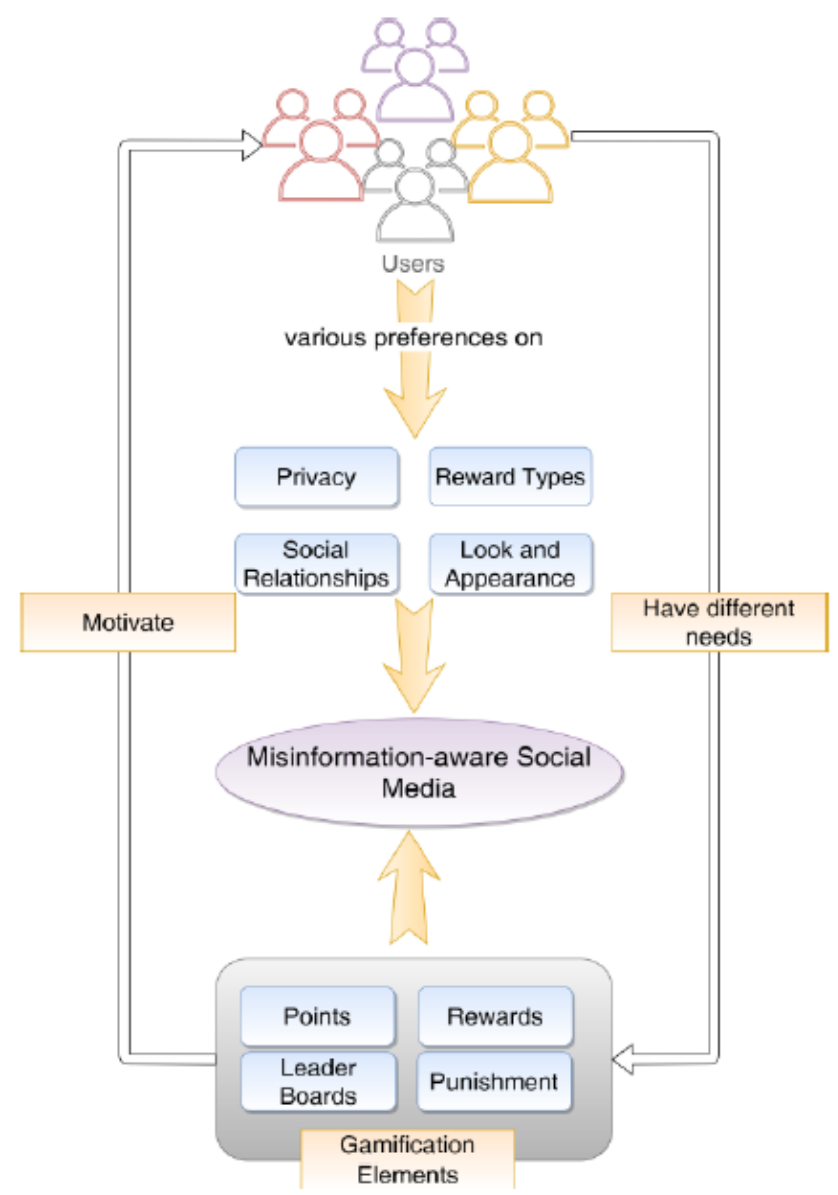

Figure 2. Conceptual framework for the design of a gamified misinformation-aware social media (Almaliki 2019)

\section{Gamified Misinformation-Aware Social Media Design Method (GMSM)}

To successfully design a Gamified Misinformation-aware Social Media (GMSM), the proposed requirement engineering method goes through three phases ordered as follows (see figure 3): Conceptual framework to scenarios; Scenarios to goal models; Goal models to use cases. These methods are well-known approaches in the domain of requirements engineering and each one of them give software engineers a better understanding and another level of details about users' various requirements and needs. GMSM combines these approaches in a sequence that can lead to a better coverage and less overlooking of important aspects of users' requirements. The following sub-section give more details about each phase and its importance in understanding users' requirements on the use of gamification design elements to minimize the distribution of misinformation on social media platforms.

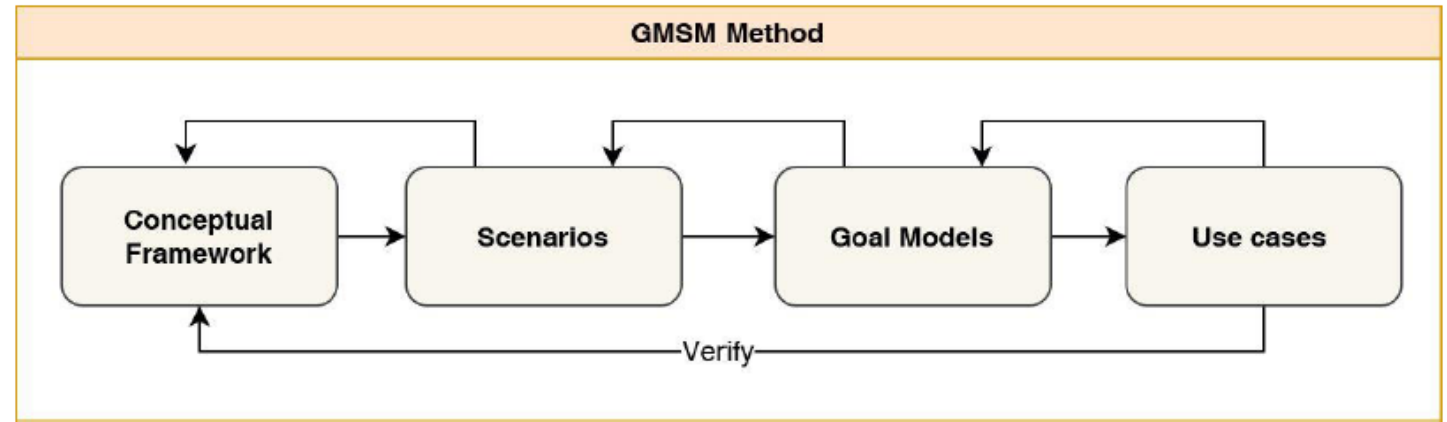

Figure 3. Gamified Misinformation-aware Social Media design method (GMSM) 


\subsection{First Phase: From Conceptual Framework to Scenarios}

In the first phase, software engineers are advised to begin the design process with authoring several scenarios based on the proposed conceptual framework (see Figure 2) following the guidelines for scenario authoring as presented in (Davis 1993, Achour 1999). Generally, a scenario has a start and final states. The start state defines a condition for the scenario to be started and the final state describes a state that is met by the end of the scenario. Additionally, a scenario is constructed of a number of actions that defines how the transition from the start state to the final state happens (Rolland, Souveyet et al. 1998). That is, scenarios should be devised in a way that respects users' behavioral needs and their variations based on the conceptual framework when applicable (e.g. the users' motivations, concerns and privacy needs).

This gives a more thorough explanation and a better understanding of the possible users' behaviors related to using of gamification to combat misinformation in different contexts (Maiden and Robertson 2005). For example, let us assume that a user called UserX (UserX represents different types of users' behaviors) uses a social media platform called MediaX to connect with friends and other people, share and post variant types of information (including news information) on a daily basis. Let us also assume that MediaX employs gamification techniques to foster better quality of news information being generated and exchanged on their platform. UserX can have several different scenarios when sharing or posting news information on MediaX in a gamified manner such as:

- Scenario 1: UserX1 shares a news post on MediaX and activates the quality rating feature of her news post to let other users rate the quality of the news being posted. UserX1 prefers to customize her privacy sittings in a way that only allows a group of her online friends to rate the news being posted. Additionally, UserX1 would prefer to know the real identity (e.g. user names) of people who rated her news posts (e.g. no nicknames or hidden names). UserX1 does not manage to find all the needed customizations on MediaX. UserX1 deactivates the quality rating feature of her news post.

- Scenario 2: UserX2 shares a news post on MediaX and activates the quality rating feature of his news post to let other users rate the quality of the news being posted. UserX2's news post was made publicly visible to all MediaX users. In addition, UserX2 included in the posts a URL to the news sources being posted. MediaX highlighted the news information being posted by UserX2 to make it different to other types of information users post (e.g. jokes, stories, etc). UsersX2 received a high number of positive ratings on the quality of the news he posted and was rewarded with a digital badge on his profile status by MediaX to acknowledge his achievement.

- Scenario 3: UserX3 shares news post on MediaX and activates the quality rating feature of his news post to let other users rate the quality of the news being posted. Before the news information was posted, a pop up that contains tips on how to verify news post is shown to UserX3 by MediaX. The tips include a link to various online fact-checking tools. UserX3 fact-checked his news information first and verify its quality then posted it. UserX3 repeats the same process before posting or sharing news information. UserX3's positive ratings on the quality of his news post increases overtime and a digital badge is rewarded to him to acknowledge that UserX3 is a trustworthy source of information.

The above scenarios can help software engineers to identify more knowledge about UserX's requirements and needs related to using gamification to fight misinformation sharing on social media platforms (e.g. MediaX). This increases the design efficiency of misinformation-aware social media which will ultimately impact users' online experience and the value of online created and exchanged content. The efficiency is based on the idea that the proposed conceptual framework (see figure 2) guides the decision regarding what should be cared for when authoring the scenario.

\subsection{Second Phase: From Scenarios to Goal Models}

One of the commonly adopted approach in the initial phases of requirement engineering is goal modelling. It enhances the effectiveness of requirement engineering process by providing modelling ways to express the logic of social and technical actors in a socio-technical system using concepts such as goals, softgoals, decomposition, actors and their interaction (Yu and Mylopoulos 1998). Intentions describes goals and goal models express the rationale of actors which indeed go along with the notion of scenarios discussed in the previous section (Faily and Fléchais 2014).

Scenarios developed in the former stage can help software engineering to help software engineers to better identify each user's (e.g. UserX) goals, softgoals and the relationship between them. This gives the ability to express the preferences between the different paths to meet goals and their impact on the goals. In general, a scenario is constructed of a number of actions that defines a unique path of the transition from the start state to 
the final state of agents (for instance UserX). Thus, scenarios can provide enough information to describe most of the behaviors (UserX's multiple scenarios) that can form the bases for developing goal models (Rolland, Souveyet et al. 1998).

This step of GMSM results in the development of a goal model that provides a richer and organized visual representation of each user's goals and the different paths that lead to reaching them. For instance, one of UserX1's drives (goals) in the first scenario is to have the quality of her news posts rated by her online friends. Nevertheless, privacy is a concern (softgoal) for UserX1 that should be catered for by at least one of the paths that leads to her goal. Otherwise, UserX1 can decline the whole idea of having her news posts rated for their quality. (see figure 4). Using scenarios as a prior step to create goal models is certainly a popular way in requirements engineering and guidelines for such process were described in (Rolland, Souveyet et al. 1998, Jones and Maiden 2005). An initial Tropos (Castro, Kolp et al. 2002, Bresciani, Perini et al. 2004) goal model that can possibly be devised from Scenario 3 that was previously introduced is shown in Figure 5.

This provides software engineers with more information about the possible alternative to reach goals and the potential impact of each alternative which will ultimately inform a successful design of a gamified misinformation-aware social media.

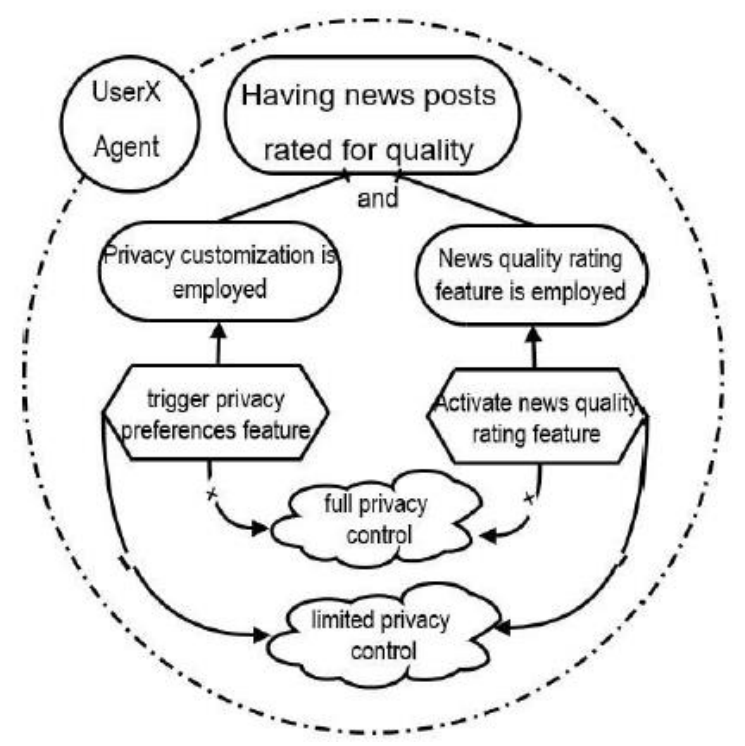

Figure 4. A potential scenario-based goal model devised from Scenario 1

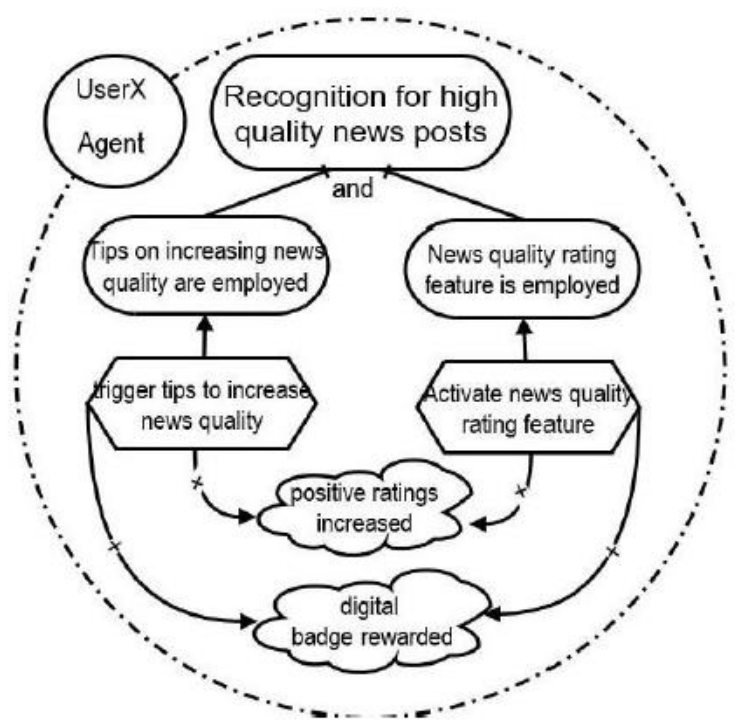

Figure 5. A potential scenario-based goal model devised from Scenario 3 
Legend
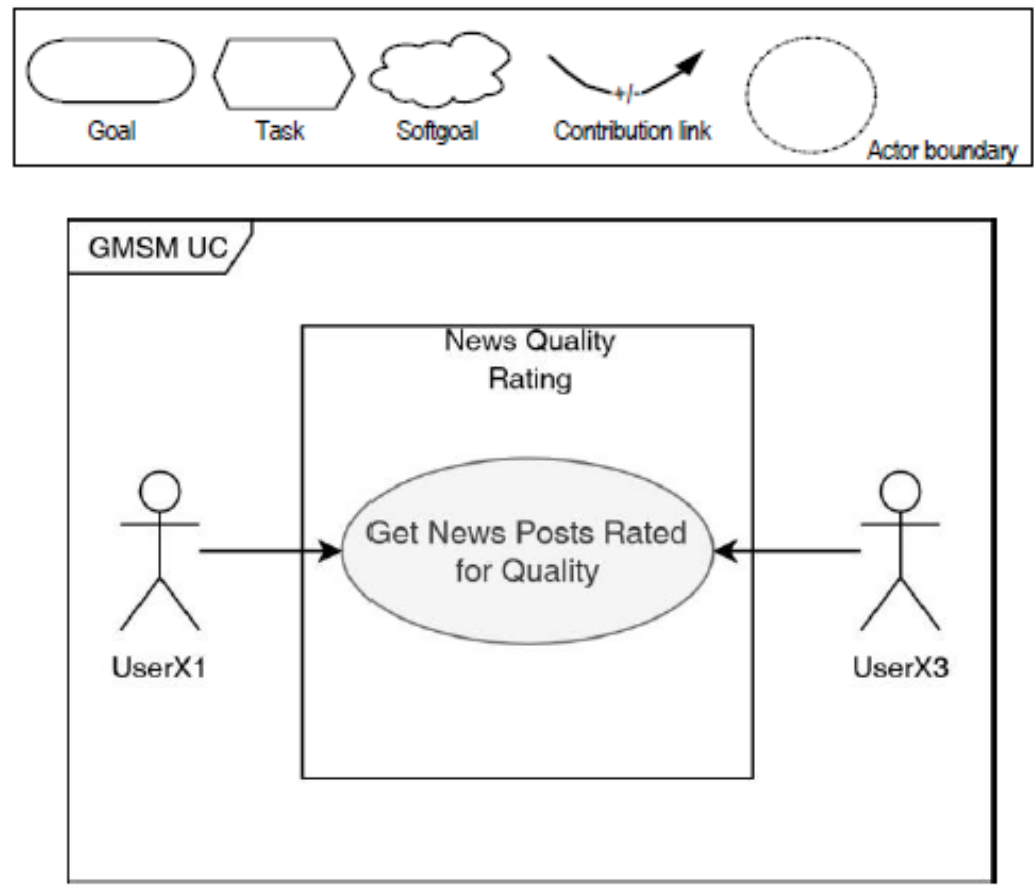

Figure 6. A goal model-based use case of UserX1 and UserX3

\subsection{Third Phase: From Goal Models to Use-Cases}

Goal modeling aims to give software engineers more knowledge and understanding of the goals and softgoals of each actor's (e.g. UserX) and expresses the various paths to meet these goals. However, goal modelling is probably not intended to express each actor's interactions (e.g. UserX) with the software. This could result in overlooking vital aspects of users' requirements. In order address this problem, the goal models created in the former stage can serve as bases for developing use-cases that express each user's interactions with the software.

To do this, one can consult the guidelines presented in (Santander and Castro 2002, Jones and Maiden 2005) which shows that joining goal models and use-cases is certainly an effective requirement engineering practice. Taking this step reduces the probability of failing to observe some important users' requirements during the design phases. For instance, UserX1 (represented in scenario 1 and goal model in figure 4), use case of Get News Posts Rated for Quality should have a flow in which full management over privacy preferences is offered to her as a primary actor (see figure 6 and table 1). However, for such a use case for UserX3 (represented in scenario 3 and goal model in figure 5), the software can act as a primary actor and autonomously perform certain actions, e.g. offering to fact-check news posts and rewarding him with a digital badge when applicable. Looking at the goal models in figure 4 and figure 5, it is possible to acquire a use case for UserX3 as shown in figure 6 and table 1.

Santander and Castro (2002) proposed an approach for the transition from goal models to use cases as presented in Figure 7. The approach focuses on goals models created based on $i^{*}$ framework (Yu 2011) and involves two key modeling approaches:

- Strategic Dependency model (SD): In this model, dependency relationships among different actors in an organizational setting are identified. It defines who an actor is and other actors who depend on the work of this actor in the context of the model.

- Strategic Rationale model (SR): In this model, a rational regarding how actors reach their goals and soft goals and a modelling of the reasons linked to actors and their dependencies are ident ${ }^{1}$ ified. The $i^{*}$ framework provide a powerful requirements engineering practice that helps software engineers better realize the problem context. The SD and SR models can next be employed to create use cases

${ }^{1}$ In (In Santander and Castro 2002) more information about the stages presented in Figure 7 can be found. 
(Santander and Castro 2002). This can lead to representing how an actor (e.g. UserX agent) interacts with the software and give a better extraction of users' preferences and requirements.

Software engineers are also advised in this phase to verify the final requirements specifications, i.e. the created goal models use cases and their narratives, against the preferences of the users as shown in the conceptual framework (see figure 2). This offers software engineers with the opportunity to minimize the possible risk of overlooking important users' requirements which can harm the achievement of effectively designing gamified misinformation-aware social media thus, the user experience and the value of online generated content.

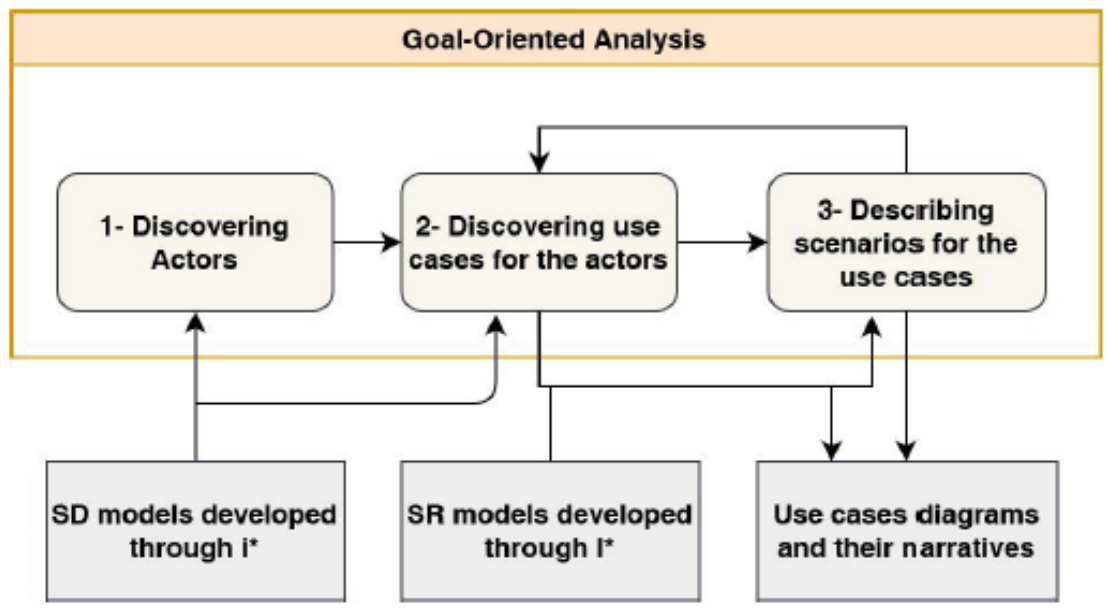

Figure $7^{1}$. Stages of the transition process from $i^{*}$ to Use Cases (Santander and Castro 2002)

Table 1. The description of the use case get news posts rated for quality

\begin{tabular}{|c|c|c|c|c|}
\hline Use Case Name & \multicolumn{4}{|c|}{ Get news posts rated for quality } \\
\hline Trigging Event & \multicolumn{4}{|c|}{ Posting news information } \\
\hline Brief Description & \multicolumn{4}{|c|}{$\begin{array}{l}\text { When a user of MediaX posts news information, a news quality rating feature is offered to the user to } \\
\text { allow other users of MediaX to rate the quality of the posted news information in a gamified manner. }\end{array}$} \\
\hline Actors & \multicolumn{4}{|c|}{ UserX1 and UserX3 } \\
\hline Preconditions & \multicolumn{4}{|c|}{ Actors visit MediaX platform, post news information and activated news quality rating feature. } \\
\hline Post conditions & \multicolumn{4}{|c|}{ Actors receive quality ratings on their news posts. } \\
\hline \multirow[t]{3}{*}{ Flow of events } & \multicolumn{2}{|c|}{ Actors } & \multicolumn{2}{|c|}{ GMSM System Response } \\
\hline & UserX3 & UserX1 & to UserX3 & to UserX1 \\
\hline & $\begin{aligned} & \text { 1- } \text { Visits MediaX } \\
& \text { Platform. } \\
& \text { 2- } \text { Logs in. } \\
& \text { 3- } \text { Clicks post to } \\
& \text { share news } \\
& \text { information. } \\
& \text { 4- } \text { Activate quality } \\
& \text { rating feature. } \\
& \text { 5- } \text { Confirm } \\
& \text { fact-checking } \\
& \text { results. } \\
& \text { 6- } \text { Confirms privacy } \\
& \text { settings. } \\
& \text { 7- } \text { Post news } \\
& \text { information. } \\
& \text { 8- } \text { Accept reward. } \\
&\end{aligned}$ & \begin{tabular}{|ll} 
1- & Visits MediaX \\
& Platform. \\
2- & Logs in. \\
3- & Clicks post to share \\
& news information. \\
4- & Activate quality \\
& rating feature. \\
$5-$ & Reject privacy \\
& settings. \\
6- & deactivate quality \\
& rating feature \\
7- & Post news \\
information.
\end{tabular} & $\begin{array}{l}\text { 2.1 Ask for log in information. } \\
\text { 3.1 Offer to activate quality } \\
\text { rating feature. } \\
\text { 4.1 Offer privacy preferences } \\
\text { customization. } \\
\text { 4.2 Offer to automatically } \\
\text { fact-check the news } \\
\text { information to be posted. } \\
\text { 7.1 Publish news post. } \\
\text { 7.2 Allow other users to rate } \\
\text { the quality of the news } \\
\text { post. } \\
\text { 7.3 Calculate the received } \\
\text { ratings accumulatively. } \\
\text { 7.4 Reward with a digital } \\
\text { badge. }\end{array}$ & $\begin{array}{l}2.1 \text { Ask for log in } \\
\text { information. } \\
\text { 3.1 Offer to } \\
\text { activate quality } \\
\text { rating feature. } \\
\text { 4.1 Offer limited } \\
\text { privacy } \\
\text { preferences } \\
\text { customization. } \\
7.1 \text { Publish news } \\
\text { post. } \\
7.2 \text { prevent other } \\
\text { users from } \\
\text { rating the } \\
\text { quality of the } \\
\text { news post. }\end{array}$ \\
\hline $\begin{array}{l}\text { Exception } \\
\text { conditions }\end{array}$ & \multicolumn{4}{|c|}{$\begin{array}{l}\text { 4.2 If the news is false after being fact-checked, UserX3 is offered to discard the post. } \\
4.2 \text { If UserX3 proceed with posting the false news, MediaX highlight the post as Fake News. } \\
7.3 \text { If more negative rating is received, UserX3 does not get rewarded. }\end{array}$} \\
\hline
\end{tabular}




\section{Conclusion}

In this paper, the authors devised a three-phase requirement engineering method for the design of Gamified Misinformation-aware Social Media (GMSM). The method is based on the recent work of the author in (Almaliki 2019) where a conceptual framework for incorporating gamification into the design of social media platform to minimize misinformation distribution was devised (see figure 2). The proposed method combines the strengths of well-known requirement engineering approaches in a sequence that can give software engineers a better understanding of users' requirements on the adoption of gamification elements to combat the distribution of misinformation on social media. This can lead to a better coverage and less overlooking of important aspects of users' requirements thus, a more satisfactory user experience and a higher quality of online shared content. In the future work, the GMSM will be enhanced with run-time adaptation abilities as users' perceptions and expectations can vary over time. That is, GMSM should be able to adapt to these changes to better match users' expectations.

\section{References}

Achour, C. B. (1999). Guiding scenario authoring. Information Modelling and Knowledge Bases, X 51, 152.

Almaliki, M. (2019). Misinformation-Aware Social Media: A Software Engineering Perspective. IEEE Access, 7 , 182451-182458. https://doi.org/10.1109/ACCESS.2019.2960270

Bresciani, P., A. Perini, P., Giorgini, F. G., \& Mylopoulos, J. (2004). Tropos: An agent-oriented software development methodology. Autonomous Agents and Multi-Agent Systems, 8(3), 203-236. https://doi.org/10.1023/B:AGNT.0000018806.20944.ef

Budak, C., Agrawal, D., \& A. El Abbadi (2011). Limiting the spread of misinformation in social networks. Proceedings of the 20th international conference on World wide web. https://doi.org/10.1145/1963405.1963499

Castro, J., Kolp, M., \& Mylopoulos, J. (2002). Towards requirements-driven information systems engineering: the Tropos project. Information systems, 27(6), 365-389. https://doi.org/10.1016/S0306-4379(02)00012-1

Chen, X. (2016). The influences of personality and motivation on the sharing of misinformation on social media. IConference 2016 Proceedings. https://doi.org/10.9776/16145

Chen, X., \& S. C. J. Sin (2013). 'Misinformation? What of it?'Motivations and individual differences in misinformation sharing on social media. Proceedings of the American Society for Information Science and Technology, 50(1), 1-4. https://doi.org/10.1002/meet.14505001102

Davis, A. M. (1993). Software requirements: objects, functions, and states, Prentice-Hall, Inc.

Deterding, S., Dixon, D., Khaled, R., \& Nacke, L. (2011). From game design elements to gamefulness: defining gamification. Proceedings of the 15th International Academic MindTrek Conference: Envisioning Future Media Environments, ACM. https://doi.org/10.1145/2181037.2181040

Duggan, M. (2017). Online harassment 2017.

Faily, S., \& Fléchais, I. (2014). Eliciting and visualising trust expectations using persona trust characteristics and goal models. Proceedings of the 6th International Workshop on Social Software Engineering, ACM. https://doi.org/10.1145/2661685.2661690

Herzig, P., Ameling, M., \& Schill, A. (2012). A generic platform for enterprise gamification. 2012 Joint Working IEEE/IFIP Conference on Software Architecture and European Conference on Software Architecture, IEEE. https://doi.org/10.1109/WICSA-ECSA.212.33

Jones, S., \& Maiden, N. (2005). Rescue: An integrated method for specifying requirements for complex sociotechnical systems. Requirements engineering for sociotechnical systems, IGI Global, 245-265. https://doi.org/10.4018/978-1-59140-506-1.ch015

Karlova, N. A., \& Fisher, K. E. (2013). A social diffusion model of misinformation and disinformation for understanding human information behaviour.

Kim, H. L., Decker, S., \& Breslin, J. G. (2010). Representing and sharing folksonomies with semantics. Journal of Information Science, 36(1), 57-72. https://doi.org/10.1177/0165551509346785

Maiden, N., \& Robertson, S. (2005). Developing use cases and scenarios in the requirements process. Proceedings of the 27th international conference on Software engineering, ACM. https://doi.org/10.1145/1062455.1062555 
Nicholson, S. (2019). A $A_{i}$ user-centered theoretical framework for meaningful gamification, 2012. Retrieved from http://scottnicholson.com/pubs/meaningfulframework.pdf

Oh, O., Agrawal, M., \& Rao, H. R. (2013). Community intelligence and social media services: A rumor theoretic analysis of tweets during social crises. MIS quarterly, 407-426. https://doi.org/10.25300/MISQ/2013/37.2.05

Ratkiewicz, J., Conover, M., Meiss, M., Gonçalves, B., Patil, S., Flammini, A., \& Menczer, F. (2010). Detecting and tracking the spread of astroturf memes in microblog streams. arXiv preprint arXiv, 1011.3768.

Rolland, C., Souveyet, C., \& Achour, C. B. (1998). Guiding goal modeling using scenarios. Software Engineering. IEEE Transactions on, 24(12), 1055-1071. https://doi.org/10.1109/32.738339

Santander, V. F., \& Castro, J. F. (2002). Deriving use cases from organizational modeling. Requirements Engineering, 2002. Proceedings. IEEE Joint International Conference on, IEEE. https://doi.org/10.1109/ICRE.2002.1048503

Starbird, K., Maddock, J., Orand, M., Achterman, P., \& Mason, R. M. (2014). Rumors, false flags, and digital vigilantes: Misinformation on twitter after the 2013 boston marathon bombing. IConference 2014 Proceedings.

Vis, F. (2014). The rapid spread of misinformation online. Outlook on the Global Agenda 2014.

Yu, E. (2011). Modelling strategic relationships for process reengineering. Social Modeling for Requirements Engineering, 11, 2011.

Yu, E., \& Mylopoulos, J. (1998). Why goal-oriented requirements engineering. Proceedings of the 4th International Workshop on Requirements Engineering: Foundations of Software Quality.

\section{Copyrights}

Copyright for this article is retained by the author(s), with first publication rights granted to the journal.

This is an open-access article distributed under the terms and conditions of the Creative Commons Attribution license (http://creativecommons.org/licenses/by/4.0/). 